\title{
Absolute Totality, Causality, and Quantum: The Problem of Metaphysics in the Critique of Pure Reason
}

\author{
Kazuhiko YAMAMOTO
}

\author{
Megumi Institute of Ethics and Philosophy, Kogashi, Japan
}

\begin{abstract}
Kant's metaphysics which says that the absolute whole of magnitude has nothing to do with any possible experience presses him to think of a thing in itself, which is merely intelligible. The difficulty is related to the issue of the absolute totality of series of conditions in connection with the issue of the absolute magnitude of the series in the world of sense, which looms as the antinomy of pure reasons. Is it possible to solve this problem in such a way that we can comprehend transcendental aesthetics and the world-whole through empirical intuition and synthesis in accordance with experience or possible experience? Our 'transcendental analytic,' grounded on the law of nature, has shown that 1) the absolute unity of the thinking subject and the absolutely unconditioned in a series of given conditions signify nullity in space-time, i.e., quantum; and 2) a being of all beings signifies space-time itself, i.e., quantum, suggesting that the understanding can never accomplish a priori anything more than to anticipate an object of experience or of possible experience. Our transcendental analytic, which is grounded on Kant's metaphysics in the Critique of Pure Reason, would potentially lead us to an alternative view of the universe in conjunction with quantum mechanics.
\end{abstract}

Keywords: Possible Experience, Absolute Totality, Causality, Quantum

\section{INTRODUCTION}

Kant, who firmly believes that the universal principles of ethics have to flow merely from his concepts of reason (A480/B508), launched an experiment which was expected to prove the pure principles of reason: "that absolutely no concepts" that "contain anything empirical" must enter into "the complete estimation of synthetic a priori cognition," or "that the a priori cognition be entirely pure" (A14/B28). However, when an intractable problem concerning "the absolute magnitude of the series in this world" (A516/B544) arises, Kant has nothing but to think that "the only thing left to us is the validity of the principle of reason as a rule for the continuation and magnitude of a possible experience, once its invalidity as a constitutive principle of appearances in themselves has been adequately demonstrated" (A516/B544). While Kant tries to adequately demonstrate the invalidity of a constitutive principle of reason, we think that he has failed. Kant has missed, unwittingly or wittingly, a crucial point, which might enable him to demonstrate its validity. So far we have clarified that: 1) in experience or in possible experience, we can ascribe sequences of an occurrence in which something happens that previously existed, to death itself - object in itself - which belongs to the category of the pure concepts of the understanding: 2) appearances themselves signify things in themselves, i.e., filled space-elapsing time or empty space-nullified time: 3) space-time itself - nullity in space-time - inheres in appearances themselves, suggesting that the standing and lasting I of pure apperception - the manifold of sensible intuition - exists in nullity in space-time, while it appears in filled space-elapsing time: 4) upon disappearance of the manifold of sensible intuition in death, filled space-elapsing time is neutralized, and it would vanish in nullity in space-time - "an a priori intuition" (B40) - returning to space-time itself: 5) for this reason, intuitions' condition belongs to one and the same series of intuitions, i.e., space-time itself: 6) "the schema of necessity" (A145) signifies space-time itself and the form of appearances in terms of 'filled-elapsing' or 'empty-nullified, which correspond to "the existence of an object at all times" (A145): 7) since a "necessary being" (A560/B588) could "occur as a condition of the existence of appearances in the world of sense" (A560/B588), a necessary being could exist as filled space-elapsing time, which appears distinct from empty space-nullified time, i.e., nullity in space-time: 8) since alterability concerns only the determinations of appearances themselves, space-time itself is the unalterable, i.e., the cause: 9) since "all appearances, considered extensively as well as intensively, are continuous magnitudes" (A171), 


\section{Kazuhiko YAMAMOTO}

"the proposition that all alteration (transition of a thing from one state into another) is also continuous" (A171-B213) is proved easily (YAMAMOTO 2016: 87-100, YAMAMOTO 2017: 1937). Furthermore, our transcendental analytic has these results: 1) all manifold of sensible intuition contains "a necessary relation" (B132), i.e., space-time itself: 2) the representation of "a necessary relation" - "pure intuition" (A21) or "an a priori intuition" (B40) - signifies space-time itself: 3) 'a necessary relation' manifests itself in the entire dissolution of the "thoroughgoing unity of selfconsciousness" (A111-A112) - death - which occurs necessarily and universally; and 4) the dissolution of the 'thoroughgoing unity of self-consciousness' is "an act of spontaneity" (B132). We have to note again that this manifold - the entire dissolution of the "thoroughgoing unity of selfconsciousness - is to be encountered in the same subject - the 'I think' - in experience or in possible experience as a result of "an act of spontaneity" (B132). Here, 'possible experience' - the possibility of death - indicates its "possibility itself" (B294), in which its "possibility as a priori cognitions of objects of an intuition in general was exhibited" (B159). Since the representation 'I think' - "the transcendental unity of self-consciousness" (B132) - is homogeneous with 'pure apperception' which signifies "the thoroughgoing identity of oneself in all possible representations" (A116) which "grounds empirical consciousness a priori" (A116), our metaphysics implies that the representation 'I think,' which can accompany all others, is to cognize "through categories whatever objects may come before our senses" (B159). In this context we comprehend that a human, as the representation 'I think,' senses, intuits and cognizes all appearances themselves in virtue of filled space-elapsing time or nullity in space-time through empirical intuition and synthesis. Thus, we rescue Kant's metaphysics from the abyss of emptiness, in which he does not comprehend something, which would be represented in him as possibility (YAMAMOTO 2017: 19-37). We have shown that 1) "the absolute unity of the thinking subject" (A335) and "the absolutely unconditioned in a series of given conditions" (A335) signify nullity in space-time, i.e., quantum; and 2) "a being of all beings" (A336) signifies space-time itself, i.e., quantum. The discourse we have made so far, which is grounded on Kant's metaphysics in the Critique of Pure Reason, would potentially lead us to an alternative view of the universe in conjunction with quantum mechanics. Therefore, we should begin to address these issues.

\section{AN A PRIORI INTUITION AND GEOMETRY}

Previously we clarified that: 1) space-time itself - nullity in space-time - is the matter, namely "that in the appearance which corresponds to sensation" (A20): 2) 'filled-elapsing' or 'empty-nullified' is "that which allows the manifold of appearance to be intuited as ordered in certain relations" (A20) (YAMAMOTO 2017: 19-37). It is clear that one, which intuits the manifold of appearance 'as ordered in certain relations,' contains "a necessary relation" (B132), i.e., the matter (YAMAMOTO 2017: 1937). We have to clarify that in what context the representation of 'a necessary relation' manifests itself as "pure intuition" (A21) or "an a priori intuition" (B40), in the entire dissolution of the "thorough going unity of self-consciousness" (A111-A112) - death - which occurs necessarily and universally. We have to note that 'an a priori intuition' can be thought to contain "an infinite set of representations within itself" (B40). Furthermore, Kant gives us a hint, saying: "Time and space are accordingly two sources of cognition, from which different synthetic cognitions can be drawn a priori,..." (B55-A39): "Those, however, who assert the absolute reality of space and time, whether they assumes it to be subsisting or only inhering, must themselves come into conflict with the principles of experience. For if they decide in favor of the first (which is generally the position of the mathematical investigators of nature), then they must assume two eternal and infinite self-subsisting non-entities (space and time), which exists (yet without there being anything real) only in order to comprehend everything real within themselves. If they adopt the second position (as do some metaphysicians of nature), and hold space and time to be relations of appearances (next to or successive to one another) that are abstracted from experience though confusedly represented in this abstraction,... and on this view the $a$ priori concepts of space and time are only creatures of the imagination, the origin of which must really be sought in experience, out of whose abstracted relations imagination has made something that, to be sure, contains what is general in them but that cannot occur without the restrictions that nature has attached to them" (B56-A40-B57). We have to pay attention to the fact that, according to Kant, "the mathematical investigators of nature' assume that 'two eternal and infinite self-subsisting non-entities (space and time)' exists 'without there being anything real,' while 'some metaphysicians of nature' other than Kant think that: 1) the a priori concepts of space and time - space itself and time itself are yielded in the imagination: 2) the origin of this imagination is to be sought in experience; and that 
3) the imagination has made something 'that cannot occur without the restrictions that nature has attached to them,' with the use of the 'abstracted relations' discerned in experience. Is there any difference between 'the mathematical investigators of nature' who assume space and time to be selfsubsisting or 'some metaphysicians of nature' other than Kant who assume them to be only inhering, while both are asserting the absolute reality of space and time? We think that they are not different but the same, on account of the fact that: 1) we can assert the reality of space and time through our perception of the real, which is to be cognized in experience or in possible experience: 2) space-time itself signifies nullity in space-time - the matter - which is homogeneous with "two eternal and infinite self-subsisting non-entities" (B56): 3) the imagination spawns a priori concepts of space and time - space-time itself - which occurs in concurrence with "the restrictions that nature has attached to them" (B57), namely death itself. Because a priori concepts of space and time - space-time itself are restricted by nature, it follows that 'two eternal and infinite self-subsisting non-entities (space and time)' is equivalent to space-time itself. As indicated above, space-time manifests itself in the entire dissolution of the 'thoroughgoing unity of self-consciousness' - death - which occurs necessarily and universally. Following our transcendental analytic, we have to think that 'two eternal and infinite selfsubsisting non-entities (space and time)' signify 'eternal and infinite self-subsisting nullity in spacetime.' Apparently, what we say here may sound contradictory on account of the fact that 'non-entity' self-subsists as nullity in space-time. However, seeing that nullity in space-time is restricted by nature, we assert, on the grounds of our transcendental analytic, that nullity in space-time signifies entity, the representation of which is "an a priori intuition" which contains "an infinite set of representations within itself" (B40). We think that "thing in itself" (A676/B704) - "a mere idea that cannot be represented in concreto at all" (A683/B711) - is to be represented, through "the restrictions that nature has attached to them" (B57), as an a priori intuition which pertains to nullity in space-time, i.e., space-time itself. We think that while an a priori intuition signifies nullity in space-time, pure intuition or empirical intuition pertains to filled space-elapsing time, implying that space-time itself, which subsists as nullity in space-time, inheres at the same time in appearance. We should like to say, in an opposite manner to what Kant refers to (A110) concerning "the restrictions that nature has attached to them" (B57), that "There is only one experience, in which all perceptions are represented as in the representation of thoroughgoing and law-like connection, just as there is only one space and time, in which all appearances and being or non-being take place. If one speaks of different experiences, they are "perceptions themselves" (B219) insofar as they belong to one and the same universal experience - death itself. The thoroughgoing and a priori unity of perceptions - nullity in space-time - is precisely what constitutes experience or possible experience, and it is nothing other than the empirical unity of the appearances in accordance with concepts.' This signifies the "empirical unity of consciousness, through association of the representations" (B139-B140), which is entirely necessary. Furthermore, in relation to this issue, we have to add, in an opposite manner to what Kant refers to (A118), saying, "the transcendental unity of apperception, whose component is "an a priori intuition" (B40), is related to the synthesis of imagination, as an a priori condition of the possibility of all composition of the manifold in a cognition. The productive synthesis of the imagination cannot take place a priori; for the productive synthesis rests on conditions of experience or possible experience. The productive synthesis of the imagination prior to apperception is the ground of the possibility of all cognition, especially that of experience or of possible experience. Now we call the synthesis of the manifold in imagination empirical if, with distinction of the intuitions, it concerns nothing but the connection of the manifold a priori, and the unity of this synthesis is called transcendental if it is represented as necessary a priori in relation to the original unity of apperception. Now since the latter is the ground of the possibility of all cognitions, the transcendental unity of the synthesis of the imagination pertains to all possible cognition, through which all objects of possible experience must be represented a priori.' This discourse indicates that "the pure (productive) synthesis of the imagination prior to apperception" (A118) need to take place a priori, as "the ground of the possibility of all cognition, especially that of experience" (A118). What is the product of "the pure (productive) synthesis of the imagination" (A118)? In Kant's metaphysics, it is nothing but his "thing in itself" (A676/B704). The product of our pure (productive) synthesis of the imagination, which, we think, takes place in conjunction with time, which could "precede the objects as a determination or order attaching to the things themselves as their condition and be cognized and intuited a priori through synthetic propositions" (A33), is nothing but nullity in space-time, i.e., the pure concept of the understanding. We think that "the transcendental unity of the synthesis of the 


\section{Kazuhiko YAMAMOTO}

imagination" (A118) signifies a human, who is represented as filled space-elapsing time, in which space-time itself - nullity in space-time - inheres. In view of the fact that filled space-elapsing time is homogeneous with empirical intuition, while nullity in space-time is with "an a priori manifold in pure intuition" (A138/B177) - an a priori intuition - it becomes clear that the "distinction of the intuitions" (A118) means the alteration of "intuitions themselves" (B160) from pure intuition to "an $a$ priori intuition,' which is to take place at the point of "the connection of the manifold a priori" (A118), i.e., at the point of the alteration from filled space-elapsing time to empty space-nullified time. Since 'death' - the pure concept of the understanding - which is yielded through the productive synthesis of the imagination, belongs, as 'the thoroughgoing and a priori unity of perceptions,' to "one and the same universal experience" (A110), we have to think that "the transcendental unity of the synthesis of the imagination" (A118), which comprises pure intuition, an a priori intuition and pure concept of the understanding - "intuitions themselves" (B160) and "perceptions themselves" (B219) - is "the ground of the possibility of all cognition" (A118), especially that of experience or of possible experience.

Seeing Kant's negative remarks toward 'the mathematical investigators of nature' and 'some meta physicians of nature,' we say that Kant's position is that 1) he does not "assume two eternal and infinite self-subsisting non-entities (space and time), which exists (yet without there being anything real)" (B56), 2) he does not "hold space and time to be relations of appearances (next to or successive to one another) that are abstracted from experience" (A40-B57). This position clearly shows us how erroneously Kant comprehends space and time. First, Kant thinks that "two eternal and infinite selfsubsisting non-entities' have nothing to do with anything real. Second, Kant thinks that "relations of appearances (next to or successive to one another) that are abstracted from experience" (A40-B57) has little relevance because they are "confusedly represented in this abstraction" (B57). What kinds of abstraction can be thought to be relevant with no confusion from Kant's viewpoint? It is the abstraction that can "occur without the restrictions that nature has attached to them" (B57). How does the abstraction occur without the restrictions that nature has attached to them? Kant has already answered it, saying "There is no other way than through concepts or through intuitions, both of which, however, are given, as such, a priori or a posteriori. The latter, namely empirical concepts, together with that on which they are grounded, empirical intuition, cannot yield any synthetic proposition except one that is also merely empirical, i.e., a proposition of experience; thus it can never contain necessity and absolute universality of the sort that is nevertheless characteristic of all propositions of geometry" (A47). On the contrary, we think that since "the transcendental unity of apperception" (A118) - "the transcendental unity of the synthesis of the imagination' - has arisen with "intuitions themselves" (B160) and "perceptions themselves" (B219), it can make a "proposition of experience' which can "contain necessity and absolute universality" (A47) - nullity in space-time - space-time itself. There are crucial differences among our 'proposition of experience,' "all propositions of geometry" (A47) and Kant's "synthetic proposition" (A47). In "all propositions of geometry" (A47), something like this could occur: "with two straight lines no space at all can be enclosed, thus no figure is possible, and try to derive it from the concept of straight lines and the number two;..." (B65). In contrast to this, in our 'proposition of experience,' an a priori intuition or pure intuition could occur with no straight lines and no number two or three. Space can be enclosed, and thus, the figure is possible here as a "synthetic proposition" (A47), which is "one that is also merely empirical, i.e., a proposition of experience" (A47). In the case of "all propositions of geometry" (A47), all of Kant's effort is in vain, forcing him "to take refuge in intuition" (B65). What does this mean? We think that, here, if nullity in space-time is introduced in accordance with an a priori intuition or pure intuition, it might neutralize the conundrum, which unavoidably occurs in "all propositions of geometry" (A47). It means that space can be enclosed with two straight lines and figure is possible, if "figures in space" (A142) are under the aegis of "pure a priori imagination" (A142). The so-called 'imaginary number' $i^{2}=-1$ in mathematics can be thought to pertain to "pure a priori imagination" (A142) or "a pure $a$ priori intuition" (A48). In our metaphysics, the 'imaginary number' $i^{2}=-1$ is commensurate with "one that is also merely empirical, i.e., a proposition of experience" (A47), which can "contain necessity and absolute universality (A47). In this regard, 'imaginary number' $i^{2}=-1$ is regarded to be 'real number,' while a 'real number' in mathematics is to be an 'imaginary number' except 'o or 1.' Kant's metaphysics is entirely different from our transcendental analytic on account of the fact that we need not use geometry or mathematics in order to have "figures in space" (A142) since "an $a$ priori intuition" (B40) or pure intuition can be thought to signify nullity in space-time - space-time 
itself - with no straight lines and no number, while Kant has to perennially deal with such issue as: "with two straight lines no space at all can be enclosed, thus no figure is possible, and try to derive it from the concept of straight lines and the number two;..." (B65). It seems that since Kant cannot tolerate the situation, he takes refuge in his "pure a priori intuition" (A48) in terms of "thing in itself" (A676/B704). We have already answered Kant's question, which says "Since the propositions of geometry are cognized synthetically a priori and with apodictic certainty, I ask: Whence do you take such propositions, and on what does our understanding rely in attaining to such absolutely necessary and universally valid truth?" (B64-A47). We have attained it through nullity in space-time, i.e., the pure concept of the understanding (YAMAMOTO 2016: 87-100, YAMAMOTO 2017: 19-37). We agree with Kant, who says that the mathematical investigators of nature and some metaphysicians of nature "can neither offer any ground for the possibility of a priori mathematical cognitions (since they lack a true and objectively valid a priori intuition), nor can they bring the propositions of experience into necessary accord with those assertions" (B57-A41). Kant, seeing that their "ground for the possibility of a priori mathematical cognitions" (B57) is "distinct from the intellectual synthesis without any imagination merely through the understanding" (B152), brings up 'thing in itself,' making his "synthetic proposition" (A47). This "thing in itself" (A676/B704) is assumed to make it possible to attain to "a priori mathematical cognitions" (B57) or "synthetic a priori cognition" (A204). We think that what Kant asserts concerning this issue, which says, "On our theory of the true constitution of these two original forms of sensibility both difficulties are remedied" (B58) is not tenable. Kant's attempt to remedy the difficulties by means of merely giving "object a priori in intuition" (A48), and grounding "synthetic proposition on this" (A48) is empty, as Kant himself repeatedly implies (A142, A147-B187, A154, A159-B199-A160, A236-B296-A237, A241-A242B300, A297, B354-A298, B380, B389, A336, A468/B496-A469/B497, A477/B505, A478/B506, A479/B507, A482/B510, A506/B534, A537/B565, A558/B586, A565/B593-A566/B594, A566/B594A567/B595, A642/B670, A644/B672-A645/B673, A644/B672, A646/B674, A647/B675, A669/B697, A676/B704, A678/B706-A679/B707, A681/B709). Kant's metaphysics is grounded on the fact that 1) "I can nevertheless assume such an incomprehensible being, the object of a mere idea, relative to the world of sense, though not in itself "(A677/B705); and 2) the "dynamical series" (A531/B559) are assumed to have "the thoroughly conditioned character" (A531/B559). Kant, in an attempt to make "the thoroughly conditioned character" appear to be "connected with a condition that is empirically unconditioned" (A531/B559), even thinks up "pure synthesis" (B104), or "synthesis, considered in itself alone" (B153), which is assumed to be capable of yielding the pure concept of the understanding or "itself determining sensibility internally" (B153). This is Kant's pure reason, but not ours. We think that "the unconditioned totality" (A531/B559) which, Kant thinks, is to arise through the synthesis of the "dynamical series' is entirely different from our "cosmological ideas dealing merely with mathematically unconditioned unity" (A532/B560) - space-time itself (nullity in space-time) which is represented by an a priori intuition or pure intuition.

\section{A Thing $=A$, Which is Something $=B$, AND CAuSAlity}

Kant makes remarks concerning "a merely logical principle," which would be proved to be crucial for directing our discourse, saying, "There is, however, still one formula of this famous principle, although denuded of all content and merely formal, which contains a synthesis that is incautiously and entirely unnecessarily mixed into it. This is: 'It is impossible for something to be and not to be at the same time.' In addition to the fact that apodictic certainty is superfluously appended to this (by means of the word 'impossible'), which must yet be understood from the proposition itself, the proposition is affected by the condition of time, and as it were says: 'A thing $=A$, which is something $=B$, cannot at the same time be non- $B$, although it can easily be both $(B$ as well as non- $B)$ in succession.'... Now the principle of contradiction, as a merely logical principle, must not limit its claims to temporal relations. Hence such a formula is entirely contrary to its aim. The misunderstanding results merely from our first abstracting a predicate of a thing from its concept and subsequently connecting its opposite with this predicate, which never yields a contradiction with the subject, but only with the predicate that is combined with it synthetically, and indeed only when both the first and the second predicate are affirmed at the same time" (A152-B192-A153). Kant, acknowledging that "no cognition can be opposed to it without annihilating itself" (B191), utters negative words toward it, saying that while this principle is a conditio sine qua non, it is not "a determining ground of the truth of our cognition" (B191-A152). He adds, saying "Since we now really have to do only with the synthetic part of our 


\section{Kazuhiko YAMAMOTO}

cognition, we will, to be sure, always be careful not to act contrary to this inviolable principle, but we cannot expect any advice from it in regard to the truth of this sort of cognition" (A152). These remarks sound astounding and ridiculous, since we think that we can expect the most important advice from it in regard to the truth of our cognition when we have to do with the empirical part of our cognition. We think that 1) all appearances themselves signify 'filled space-elapsing time' or 'empty space-nullified time,' 2) 'filled space-elapsing time' cannot at the same time be 'empty space-nullified time.' Therefore, we would say that such a proposition as "A thing $=A$, which is something $=B$, cannot at the same time be non- $B$ " should be formulated such as that "appearance itself = appearance, which is filled space-elapsing time, cannot at the same time be non-appearance itself, i.e., empty space-nullified time.' Since empty space-nullified time is commensurate with nullity in space-time, which permeates 'appearance itself = appearance,' nullity in space-time - space-time itself - can at the same time be 'appearance itself = appearance,' without being affected by the condition of time. Therefore, we would say, 'It is possible for something to be and not to be at the same time,' and 'A thing $=A$, which is something $=B$, can at the same time be non- $B$.' Of course it is axiomatic that: 1) it is impossible for a part of filled space-elapsing time and another part of filled space-elapsing time to be at the same point in the same instance, 2) it is possible for a part of empty space-nullified time and another part of empty space-nullified time to be at the same point in the same instance - nullity in space-time - if points and instances are conjured up in nullity in space-time.

In relation to this issue, it is critically important for us to clarify how we cognize, through empirical intuition and "synthesis of apprehension" (B164), the transition of space-time itself from filled spaceelapsing time to empty space-nullified time and how we cognize the space-time itself on the grounds for the "constitutive principle of reason" (A509/B537). In regard to this principle, we have to say, in an opposite manner to what Kant says (A509/B537), that it is 'a principle of the greatest continuation and extension of possible experience, in accordance with which empirical boundary as nullity in space-time would hold as an absolute boundary.' Though a part of the discourse has been already made (YAMAMOTO 2016: 87-100, YAMAMOTO 2017: 19-37), we will deal with these issues again in an attempt to make it more explicit. When Kant makes remarks in regard to "how in general anything can be altered" (B252), saying "how it is possible that upon a state in one point of time an opposite one could follow in the next - of these we have a priori not the least concept. For this acquaintance with actual forces is required, which can only be given empirically, e.g., acquaintance with moving forces, or, what comes to the same thing, with certain successive appearances (as motions) which indicate such forces. But the form of such an alteration, the condition under which alone it, as the arising of another state, can occur (whatever the content, i.e., the state, that is altered might be), consequently the succession of the states itself (that which has happened), can still be considered a priori according to the law of causality and the conditions of time" (B252-A207), we agree with Kant. We have already made a discourse concerning the origin of humanity, saying: 'we have to take note that 'empirical intuition' does not necessarily correspond to 'empirical intuition and synthesis,' on account of the fact that synthesis signifies "an act of the spontaneity of the power of representation," "in distinction from sensibility" (B130). We think that this act of the spontaneity of the power of representation, in distinction from sensibility, signifies the origin of humanity itself' (YAMAMOTO 2017: 19-37): 'When the standing and lasting I of pure apperception could acquire the concept of death - the pure concepts of understanding - through empirical intuition and synthesis of apprehension, upon encountering the phenomena of disappearance, it emerged as a human. In other words, when it cognized nullity in space-time, which already resides in them, it emerged in the representation of space-time itself, and began to exist as a human" (YAMAMOTO 2017: 19-37). We think that once a living thing could arise as a human through the acquaintance with actual forces, i.e., "realitates phaenomena" (B329), he or she would be able to transmit the pure concepts of the understanding to their posterity with language, and teach it as phaenomena with "the unity of the categories" (A249). Therefore, we can say that, of "how in general anything can be altered" (B252), we have ample concepts, which are given a priori.

In regard to the alteration and causality, we have clarified that 1) all alteration as a transition of a thing from one state to another signifies the transition from a state of appearance to a state of disappearance, or from a state of disappearance to a state of appearance, and 2) the form of appearance, which alterability concerns, is 'filled-elapsing' or 'empty-nullified,' while their cause is in the unalterable space-time itself (YAMAMOTO 2017: 19-37). In regard to alteration and causality, Kant has made an enigmatic and contradictory discourse, saying "If a substance passes out of a state $a$ into another state 
$b$, then the point in time of the latter is different from the point in time of the first state and follows it. Likewise the second state as a reality (in the appearance) is also distinguished from the first, in which it did not yet exist, as $b$ is distinguished from zero; i.e., if the state $b$ differs from the state $a$ even only in magnitude, then the alteration would be an arising of $b-a$, which did not exist in the prior state, and with regard to which the latter $=0 "$ " (B253-A208). On the grounds of what we have already clarified, we think that Kant's statement indicates four things: 1) a state $a$ signifies space-time itself, while another state $b$ signifies filled space-elapsing time: 2 ) the second state - filled space-elapsing time - as a reality in the appearance, should be distinguished from the first - space-time itself - as a reality in nullity in space-time: 3 ) intensive magnitude of the state $b$ is distinguished from zero, while the state $a$ has intensive magnitude, which is zero: 4) the state $b$ and the state $a$ have extensive magnitude "in which the representation of the parts makes possible the representation of the whole" (A162). Thinking like that, we entirely agree with what Kant refers to here. In this context, it comes out that space-time itself - the cause which is unalterable - spawns the transition from a state of disappearance to a state of appearance, which is correspondent to the transition of space-time itself from 'empty-nullified' to 'filled-elapsing.' This is not contradictory on account of the fact that since space-time itself permeates filled space-elapsing time, the unalterable always inheres. Here, Kant seems to be making a discourse concerning only the arising of the manifold of appearance in the world of sense from nullity in space-time. In addition, we would say that we have a concept of causality in regard to a transition of space-time itself from 'filled-elapsing' to 'empty-nullified.' We have an "acquaintance with actual forces... which can only be given empirically" (A207), or "what comes to the same thing, with certain successive appearances (as motions)" (A207). Therefore, we can draw from this acquaintance, the concept of causality in terms of "how it is possible that upon a state in one point of time an opposite one could follow in the next" (B252-A207), which is correspondent to a transition of space-time itself from 'filled-elapsing' to 'empty-nullified.' Furthermore, there could be additional causality, which is, according to Kant, "the form of such an alteration, the condition under which alone it, as the arising of another state, can occur ..., consequently the succession of the states itself (that which has happened), can still be considered $a$ priori according to the law of causality and the conditions of time" (A207). Therefore, here, we have to make a discourse about causality.

Kant again gives us a hint for the direction of discourse, which could enable us to comprehend, in transcendental analytic, the causality. It says, "In respect of what happens, one can think of causality in only two ways: either according to nature or from freedom. The first is the connection of a state with a preceding one in the world of sense upon which that state follows according to a rule. Now since the causality of appearances rests on temporal conditions, and the preceding state, if it always existed, could not have produced any effect that first arose in time, the causality of the cause of what happens or arises has also arisen, and according to the principle of understanding it in turn needs a cause. By freedom in the cosmological sense, on the contrary, I understand the faculty of beginning a state from itself, the causality of which does not in turn stand under another cause determining it in time in accordance with the law of nature" (A532/B560-A533/B561). We think that when a substance passes out of a state $a$ into another state $b$, there are three modes of alteration: 1) an alteration of a part of filled space-elapsing time to empty space-nullified time: 2) a passing out of a part of filled spaceelapsing time into a part of filled space-elapsing time as "the succession of the states itself" (A207): 3) an alteration of empty space-nullified time to a part of filled space-elapsing time. It is clear that "the causality of appearances" in these alterations or a passing out stands under temporal conditions. In the alteration of empty space-nullified time to a part of filled space-elapsing time, the preceding state could not have produced any effect that first arose in time since it is always nullity in space-time. This means that "the causality of the cause of what happens or arises" (A532/B560) is not necessary, indicating that, according to Kant's principle of understanding, it does not have a cause. On the contrary, according to our principle of understanding, it needs not a cause since space-time itself, namely nullity in space-time is the cause itself. Therefore, we should say that "the causality of appearances" (A532/B560) is commensurate with "beginning a state from itself" (A533/B561). In other words, the manifold of appearance would arise in "a spontaneity, which could start to act from itself, without needing to be preceded by any other cause that in turn determines it to action according to the law of causal connection" (A533/B561).

Then we have to clarify 'the causality of disappearances' and the causality of "the succession of the states itself" (A207). Again, Kant gives us a hint, saying, "For according to the principle of causality 


\section{Kazuhiko YAMAMOTO}

actions are always the primary ground of all change of appearances, and therefore cannot lie in a subject that itself changes, since otherwise further actions and another subject, which determines this change, would be required. Now on this account action, as a sufficient empirical criterion, proves substantiality without it being necessary for me first to seek out its persistence through compared perceptions, a way in which the completeness that is requisite for the quantity and strict universality of the concept could not be attained. For that the primary subject of the causality of all arising and perishing cannot itself arise and perish (in the field of appearances) is a certain inference, which leads to empirical necessity and persistence in existence, consequently to the concept of a substance as appearance" (A205-B251-A206). Here Kant has candidly confessed that he cannot seek out the persistence of action "through compared perceptions" (B251), indicating that he has missed the crucial one - perishing "in the field of appearances" (B251). In the case of living things, 'perishing' is equivalent to death itself. From our viewpoint, this enigmatic remark indicates five things: 1) while it is clear that "actions are always the primary ground of all change of appearances" (A205), they always come to a part of filled space-elapsing time from another part of filled space-elapsing time: 2) since it cannot "lie in a subject that itself changes" (A205), "further actions and another subject" (A205) - another part of filled space-elapsing time - which determines this change, would be required: 3) "through compared perceptions" (B251), it is possible to attain "the completeness that is requisite for the quantity and strict universality of the concept" (B251): 4) since "the primary subject of the causality of all arising and perishing" (B251) corresponds to space-time itself, it "cannot itself arise and perish" (B251): 5) since "empirical necessity and persistence in existence" (B251) signify filled space-elapsing time or empty space-nullified time, it leads "consequently to the concept of a substance as appearance" (A206) - space-time itself. Therefore, when Kant says, "Now there is no existence that could be cognized as necessary under the condition of other given appearances except the existence of effects from given causes in accordance with laws of causality. Thus it is not the existence of things (substances) but of their state of which alone we can cognize the necessity, and moreover only from other states, which are given in perception, in accordance with empirical laws of causality" (A227-B280), we entirely agree with him, thinking that 1) the "existence that could be cognized as necessary under the condition of other given appearances" (A227) corresponds to "effects from given causes in accordance with laws of causality" (A227): 2) "the existence of effects" (A227) is cognized as necessary in the alteration of a part of filled space-elapsing time to empty spacenullified time through empirical intuition and synthesis of apprehension: 3 ) effects come as a result of alteration of "states, that are given in perception" (B280) - the alteration of a part of filled spaceelapsing time to empty space-nullified time or the alteration of empty space-nullified time to a part of filled space-elapsing time. Kant has already shown us a direction to proceed in order to attain the concept of causality, saying, "The schema of the cause and of the causality of a thing in general is the real upon which, whenever it is posited, something else always follows. It therefore consists in the succession of the manifold insofar as it is subject to a rule" (A144): "Necessity therefore concerns only the relations of appearances in accordance with the dynamical law of causality, and the possibility grounded upon it of inferring a priori from some given existence (a cause) to another existence (the effect). Everything that happens is hypothetically necessary; that is a principle that subjects alteration in the world to a law, i.e., a rule of necessary existence, without which not even nature itself would obtain" (B280-A228). Acknowledging Kant's admonition in regard to the issue of causality, which says, "we cognize only the necessity of effects in nature, the cause of which are given to us, and the mark of necessity in existence does not reach beyond the field of possible experience,..." (B280), we would begin to address this issue, thinking, in opposition to what Kant says (B280), that 'in this it does hold of the existence of things, as substances, since these can be regarded as empirical effects, or as something that happens and arises.'

In regard to "the schema of the cause and of the causality of a thing in general...the real" (A144), we have already clarified that 1) perception, hence experience, is possible that would prove an entire presence of everything real in disappearance, i.e., a proof of empty space or of empty time can be drawn from experience or from possible experience: 2) the entire presence of the real in disappearance can be perceived in possible experience of death, and, it can be deduced from any single disappearance, from the difference in the degree of reality: 3) thus, humans perceive "the real, which corresponds to sensations in general, in opposition to the negation $=\mathrm{o}$ " (B217) or in conformity with the negation $=0: 4)$ appearances themselves, which signify filled space-elapsing time or nullity in space-time that is given a priori, constitutes "the sensation (as matter of perception)" (A167-B209) quantum (YAMAMOTO 2017: 19-37). Thus, we have clarified that "the schema of the cause and of 
the causality of a thing in general" (A144) grounds in "realities in appearance (realitas phaenomenon)" (A265), which "can certainly be in opposition with each other and, united in the same subject, one can partly or wholly destroy the consequence of the other" (B321). Then we have to clarify the necessary "relations of appearances in accordance with the dynamical law of causality" (B280-A228) - "everything that happens is hypothetically necessary" (A228). Kant provides us an invaluable and indispensable hint, which would enable us to penetrate into the causality, thereby putting it on the grounds of the constitutive principle of reason. Accordingly, Kant says, "Now I call that magnitude which can only be apprehended as a unity, and in which multiplicity can only be represented through approximation to negation $=0$, intensive magnitude. Thus every reality in the appearance has intensive magnitude, i.e., a degree. If one regards this reality as cause (whether of the sensation or of another reality in appearance, e.g., an alteration), then one calls the degree of reality as cause a 'moment,' e.g., the moment of gravity, because, indeed, the degree designates only that magnitude the apprehension of which is not successive but instantaneous" (B210-A169). From our viewpoint, this enigmatic remark indicates six things: 1) the world-whole - a unity - signifies magnitude: 2) this world-whole - magnitude - is commensurate with space-time itself, namely nullity in space-time, in which multiplicity can have intensive magnitude, which makes them approximate to negation $=0: 3$ ) nullity in space-time pertains to "every reality in the appearance" - multiplicity in the world-whole: 4) intensive magnitude, which is making an approximation to negation $=0-$ nullity in space-time - signifies "the moment of gravity," which is the cause: 5) intensive magnitude - a degree - is to be apprehended instantaneously upon the dissolution of a part of filled space-elapsing time - a diminution of the multiplicity: 6) magnitude signifies extensive magnitude, cognized as nullity in space-time in accordance with experience of death or its "possibility itself" (B294), or intensive magnitude - a degree - which is to be apprehended instantaneously in an alteration of "the sensation (as matter of perception)" (A167-B209) or of the "realities in appearance (realitas phaenomenon)" (A265). The result of the transcendental analytic corresponds to the aforementioned discourse that nullity in space-time - space-time itself - permeates filled space-elapsing time as a cause.

Then, following the scheme in the "Table of Categories" (B106), which ordains that "allness (totality) is nothing other than plurality considered as a unity, limitation is nothing other than reality combined with negation, community is the causality of a substance in the reciprocal determination of others, finally necessity is nothing other than the existence that is given by possibility itself" (B111), we have to deal with the issue of community (reciprocity) in 'allness (totality),' i.e., 'plurality considered as a unity' since "The schema of community (reciprocity), or of the reciprocal causality of substances with regard to their accidents, is the simultaneity of the determinations of the one with those of the other, in accordance with a general rule" (A144-B184). Kant's discourse would lead us to an astonishing finding in regard to the issue of causality. Accordingly he says, "since the parts of space are not subordinated to one another but are coordinated with one another, one part is not the condition of the possibility of another, and space, unlike time, does not in itself constitute a series. Yet the synthesis of the manifold parts of space, through which we apprehend it, is nevertheless successive, and thus occurs in time and contains a series" (B439). From our viewpoint, this enigmatic remark indicates seven things: 1) since there are no parts of space in space itself - nullity in space - space, as space itself, is not "subordinated to one another" and are not "coordinated with one another" (B439): 2) therefore, space, as space itself, does not constitute a series: 3) since there are 'filled spaces' as manifold parts of space itself, the manifold parts of space itself are subordinated to one another or are coordinated with one another: 4) therefore, a 'filled space' - a manifold part - can be the condition of the possibility of another part, and 'filled space,' like 'elapsing time,' does in itself constitute a series: 5 ) the synthesis of the manifold part of space itself (synthesis of 'filled space' or of a 'filled space' and empty space) is "successive, and thus occurs in time and contains a series" (B439): 6) since the synthesis takes place in the manifold of sensibility, 'succession,' 'subordination' and 'coordination' pertain to filled space-elapsing time: 7) since the world-whole consists of all appearances - filled space-elapsing time and nullity in space-time - 'succession,' 'subordination' and 'coordination' which take place in filled space-elapsing time would affect the world-whole. Following what Kant says (B112), contrarily, we have to say, "Now a similar connection is thought of in an entirety of things, since one is subordinated, as effect, under another, as the cause of its existence, or coordinated with the other simultaneously and reciprocally as cause with regard to its determination.' What does this mean? It means that "the members of the division exclude each other and yet are connected in one sphere, so in the latter case the parts are represented as ones to which existence (as substances) pertains to each exclusively of the others, and which are yet connected in one whole" (B113). We 
think that "the members of the division" signifies categories, through which it would become possible for us to cognize "whatever objects may come before our senses, ... as far as laws of their combination are concerned" (B159). When Einstein asserts that there is a serious defect in quantum mechanics (Einstein et al. 1935: 777-780), we feel that our transcendental analytic, grounded on Kant's metaphysics in the Critique of Pure Reason, might give us an inkling into the solution of this conundrum. This issue will be addressed in our following discourse.

\section{REFERENCES}

Einstein, Albert, Podolsky, Boris, Rosen, Nathan, "Can Quantum-Mechanical Description of Physical Reality Be Considered Complete?," Physical Review 47: 777-780, 1935.

Kant, Immanuel, Critique of Pure Reason, Cambridge University Press, 1999.

Yamamoto, Kazuhiko, "The Pure Concepts of the Understanding and Synthetic A Priori Cognition: the Problem of Metaphysics in the Critique of Pure Reason and a Solution," Proceedings of the European Conference on Ethics, Religion \& Philosophy 2016, pp 87-100, 2016.

Yamamoto, Kazuhiko, "The Transcendental Aesthetic and Absolute Totality of Conditions: The Problem of Metaphysics in the Critique of Pure Reason and a Solution," International Journal of Humanities Social Sciences and Education 4 (2): 19-37, 2017. 